\title{
The multiplicity of particle production from hadron- hadron and nucleus-nucleus interaction
}

\author{
Ahmed Abdo Ahmed Al-Haydari ${ }^{1}$, Mohmmed Tarek Hussein ${ }^{2}$ \\ ${ }^{1}$ Physics Department, Faculty of Applied Science Taiz University, Taiz, Yemen; a alhaydari@yahoo.com \\ ${ }^{2}$ Physics Department, Faculty of Science, Cairo University, Giza, Egypt; hussein1@mailer.eun.eg
}

Received 21 October 2009; revised 26 November 2009; accepted 23 February 2010.

\begin{abstract}
The particle production in hadron-nucleon $(\mathrm{h}-\mathrm{N})$, hadron-nucleus $(h-A)$ and nucleus-nucleus ( $A-A)$ collisions at high energies are studied in view of the multi-peripheral model. A multi-peripheral T-matrix element is assumed with multi surface parameter that is functionally dependent on the number of particles in the final state and control the kinematical path of the reaction. A Monte Carlo code is designed to simulate events according to a hypothetical model, the quark structure of the interacting nucleons is considered. The number of possible nucleon collisions inside the target nucleus plays an important role in folding the (h-N) to generate the (h-A) and (A-A) collisions. The predictions of the model give reasonable agreement with the recently examined experimental data.
\end{abstract}

Keywords: Monte-Carlo Generators; Multiplicity Distribution; Integral Phase Space

\section{INTRODUCTION}

In the last few years, research work has been concentrated on the possible existence of the quark-gluon plasma phase, considering of unconfined quarks and gluons at high temperature or high density. In the laboratory, nucleus-nucleus collisions at very high energies provide a promising way to produce high temperature or high-density matter. As estimated by Bajorken [1] the energy density can be so high that these reactions might be utilized to explore the existence of the quark-gluon plasma. One of the many factors that lead to an optimistic assessment that matter at high density and high temperature may be produced with nucleus-nucleus collisions is the occurrence of multiple collisions. By this we mean, a nucleon of one nucleus may collide with many nucleons in the other and deposit a large amount of energy in the collision region. In the nucleon-nucleon cen- ter of mass system, the longitudinal inter-nucleon spacing between target nucleons is Lorentz contracted and can be smaller than $1 \mathrm{fm}$ in high-energy collisions. On the other hand, particle production occurs only when a minimum distance of about $1 \mathrm{fm}$ separates the leading quark and antiquark in the nucleon-nucleon center of mass system [2,3]. Therefore when the projectile nucleon collides with many target nucleons, particle production arising from the first N-N collision is not finished before the collision of the projectile with another target nucleon begins. There are models [4-11] that describe how the second collision is affected by the first one. Nevertheless, the fundamental theory of doing that remains one of the unsolved problems. Experimental data suggest that after the projectile nucleon makes a collision, the projectile-like object that emerges from the first collision appears to continue to collide with other nucleons in the target nucleus on its way through the target nucleus. In each collision the object that emerges along the projectile nucleon direction has a net baryon number of unity because of the conservation of the baryon number. One can speak loosely of this object as the projectile or baryon-like object and can describe the multiple collision process in terms of the projectile nucleon making many collisions with the target nucleons. Then, losing energy and momentum in the process, and emerging from the other side of the target with a much diminished energy. The number of collisions depends on the thickness of the target nucleus. Experimental evidence of occurrence of multiple collision process can be best illustrated with the data of $p-A$ reactions in the projectile fragmentation region $[3,12]$. In the present work, we shall investigate the particle production mechanism in heavy ion collision by introducing the multi- peripheral model $[13-16,18]$ which is based on the phase space integral to describe the multi-particle production in the Hadron-Hadron, Hadron-Nucleus and Nucleus-Nucleus interaction at different energies. In this technique the many body system is expanded into subsystems, each one concerns a two body collision where we have used the matrix element of the multi-peripheral nature whose 
parameters are strongly correlated to the final state multiplicity. The simplified quark-quark interaction picture is considered to improve the results; we suppose that all the quarks which constitute the hadrons contribute in the reaction. It is assumed that each Hadron in the final state is produced at the specific peripheral surface that is characterized by a peripheral parameter.

\section{THE MULTI-PERIPHERAL MODEL}

We start with the initial single state of center of mass energy $\sqrt{s}$, let us denote by $t_{i}$ the square of the 4-vectore momentum transferred from the particle $p_{a}$ of mass $m_{\mathrm{a}}$ to the system of 4-momenta $k_{n-1}$ with mass $M_{n-1}$. This will reduce the many body problem into $n-1$ iterative diagram, each of them has only two particles in the final state. For example, the $i^{\text {th }}$ diagram has two in the final state, the first one is the particle number $n-1$, and the other has an effective mass $M_{i}$, equivalent to the rest of the $i$ particles of the system. The general expression for $t_{i}$ in the center of mass frame of $K_{i+1}$ is Kinematically calculated as,

$$
\begin{aligned}
& t_{i}=\left(p_{a}-k_{i}\right)^{2}=m a^{2}+M_{i}^{2}-2 E_{a}{ }^{(i+1)} k_{i}^{0} \\
& +2 P_{a}^{(i+1)} K_{i} \cos \theta_{i}
\end{aligned}
$$

where $K_{i}$ and $P^{(i)}$ are the magnitude of the 3-vector momentum of a system of $i$ particles and the $i^{t h}$ splitted one, $\theta_{i}$ and $k_{i}^{0}$ are the scattering angle and the energy of the system $M_{i}$. The recursion relations of $P^{(i)}$ and $K_{i}$ are given by,

$$
\begin{aligned}
& K_{i}=\frac{\sqrt{\lambda\left(M^{2}{ }_{i+1}, M_{i i}^{2}, m^{2}{ }_{i+1}\right)}}{2 M_{i+1}} \\
& P_{a}^{(i)}=\frac{\sqrt{\lambda\left(M^{2}{ }_{i}, t_{i}, m^{2}{ }_{a}\right)}}{2 M_{i}}
\end{aligned}
$$

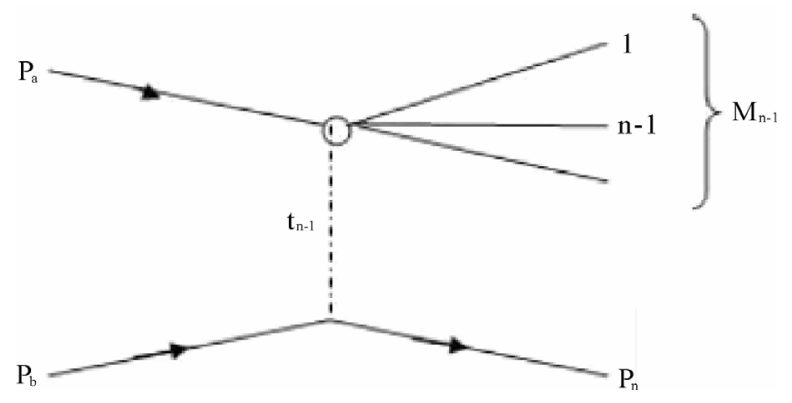

Figure 1. The basic process diagram of $p_{a}+p_{b}=K_{n-1}+p_{n}$ expressed as a sequence of two particles decay. and the corresponding energies $k_{i}^{0}$ and $E_{a}^{(i)}$ of the system $M_{i}$ are given by,

$$
\begin{aligned}
& k_{i}^{0}=\left(M_{i}^{2}+K_{i}^{2}\right)^{\frac{1}{2}}, k_{n}^{0}=M_{n}=\sqrt{s} \\
& E_{a}^{(i)}=\left(P_{a}^{(i) 2}+m_{a}{ }^{2}\right)^{1 / 2}
\end{aligned}
$$

For the case where $i=n$, we get

$$
P_{a}^{(n)}=\frac{\sqrt{\lambda\left(s, m_{b}{ }^{2}, m_{a}^{2}\right)}}{2 \sqrt{s}}
$$

where $m_{a}$ and $m_{b}$ are the masses of the initially interacting particles. The function denotes the Lorentz invariant function which is defined by,

$$
\lambda(x, y, z)=(x-y-z)^{2}-4 y z
$$

The phase space integral $I_{n}(S)$ which expresses the probability of obtaining a final state of n-particles with total center of mass (C. M) $\sqrt{s}$ in which energy and momentum are conserved is given by,

$$
I_{n}(s)=\int \ldots \ldots \ldots . \int d^{3} p_{i} / 2 E_{i} \delta^{4}\left(p_{a}-p_{b}-\sum p_{i}\right)\left|T\left(P_{i}\right)\right|^{2}
$$

where $T\left(p_{i}\right)$ is the transition matrix element that represents the transition probability from an initial state $p_{a}+p_{b}$ to the final state $K_{n-1}+P_{n-1}$ with the definite momentum $p_{i}$. Once the phase space integral is defined, one can easily find the reaction cross-section as

$$
\sigma_{n}=(1 / F) I_{n}(s)
$$

where $F$ is the flux function defined by,

$$
F=2 \sqrt{\lambda\left(s, m_{b}^{2}, m_{a}^{2}\right)}(2 \pi)^{2 n-4}
$$

If $x=x\left(p_{i}\right)$ is any physical quantity depending on the $p_{i}$, the differential cross-section $\frac{d \sigma_{n}}{d x}$ is obtained by transforming the integral in Eq.8 so that $x$ appears as a variable and then omitting the integration over $x$. This can be most simply carried out by inserting the constraint $x=x\left(p_{i}\right)$ in the integrand as a $\delta$ function so that

$$
\begin{aligned}
& \frac{d \sigma_{n}}{d x}=(1 / F) \int \ldots \ldots . \int \frac{d^{3} p_{i}}{2 E_{i}} \delta^{4}\left(p_{a}+p_{b}-\sum p_{i}\right) \delta^{4} \times \\
& \left(x-x\left(p_{i}\right)\right)\left|T\left(p_{i}\right)\right|^{2}
\end{aligned}
$$

The multiperipheral matrix element [17] is introduced in the form,

$$
T=\prod_{i=1}^{n-1} g_{i}\left(t_{i}\right)
$$


The function $g_{i}\left(t_{i}\right)$ cuts of large values of $t_{i}$ for instance one may choose

$$
g_{i}\left(t_{i}\right)=\exp \left(-a_{i} t_{i}\right)
$$

where $a_{i}$ is a peripheral parameter that play an important role in converging the particles in phase space and consequently, control the energy of the particles in final state. So that the values of $a_{i}$ are adjusted to conserve the total energy. The energy $E^{(i)}$ of the particle number $i$ is related to its rapidity $y_{i}$ through the relation,

$$
E^{(i)}=m_{t}=\cosh \left(y_{i}\right)
$$

where $m_{t}$ is defined by,

$$
m_{t}=\sqrt{P_{t}^{2}+m_{i}^{2}}
$$

so that the total energy of the particles in the final state is

$$
\zeta_{i}^{n}=\frac{1}{\sigma_{n}} \int m_{t} \cosh (y)(d \sigma / d y) d y
$$

$\zeta_{i}^{n}$, is the function of the parameters $a_{i}$ which should be compared with the total center of mass energy $\sqrt{s}$ of the initial state. We first start with $n=1$ to get $a_{1}$, which is inserted again in the case $n=3$ to get $a_{2}$ and so on. These are repeated sequentially to get the values of the rest parameters. The integral phase space Eq.8 is then calculated as,

$$
\begin{aligned}
& I_{n}(s)=\frac{1}{2 M_{n}} \cdot \frac{1}{4 P_{a}^{(n)}} \int_{\mu n-1}^{M_{n}-m_{n}} d M_{n-1} \int_{t^{-}{ }_{n-1}}^{t^{+}} \exp \left(a_{n-1} t_{n-1}\right) d t_{n-1} \int_{0}^{2 \pi} d \phi_{n-1} \ldots \ldots \ldots \ldots . . . \\
& \ldots \ldots \ldots . . . \frac{1}{4 P_{a}^{(3)}} \int_{\mu 2}^{M_{3}-m_{3}} d M_{2} \int_{t_{2}{ }^{-}}^{t_{2}{ }^{+}} \exp \left(a_{2} t_{2}\right) d t_{2} \int_{0}^{2 \pi} d \phi_{2} \frac{1}{4 p_{a}^{(2)}} \int_{t_{1}{ }^{-}}^{t_{1}{ }^{+}} \exp \left(a_{1} t_{1}\right) d t_{1} \int_{0}^{2 \pi} d \phi_{1}
\end{aligned}
$$

The multiple integration in Eq.16 may be solved by using the Monte-Carlo technique [21-23]. At extremely high energy, Eq.16 has an asymptotic limit in the form,

$$
I_{n}(s)=\frac{1}{2 \sqrt{s}} \prod\left\{\frac{\pi}{2 P_{a}^{(i+1)}} \frac{e^{a_{i} t_{i}^{+}}-e^{a_{i} t_{i}^{-}}}{a_{i} e^{a_{i} t_{i}}}\right\} \frac{\left(\sqrt{s}-\mu_{n}\right)^{n-2}}{(n-2) !}
$$

where $\left\{\frac{e^{a_{i} t_{i}^{+}}-e^{a_{i} t_{i}^{-}}}{a_{i} e^{a_{i} t_{i}}}\right\}$ is the normalization density and $\mu_{n}$ is defined by,

$$
\mu_{i}=\sum_{j} m_{j}, j=1, \ldots \ldots i
$$

Let $r^{(i)}$ be a group of $i$-random numbers, $0 \leq r^{(i)} \leq 1$, then the invariant mass $M_{i}$ for a system of $i$-particles can be generated according to

$$
M_{i}=\mu_{i}+r^{(i)}\left(M_{i+1}-\mu_{i}\right)
$$

It means that, the invariant masses vary between the limits $\mu_{i} \leq M_{i} \leq M_{i+1}-m_{i+1}, i=2, \ldots, n-1$ for the special case where $T$ is constant (no dynamical effect), the momentum transfer $t_{i}$ should vary homogeneously between the two limiting values $t_{i}^{ \pm}$,

$$
t_{i}^{ \pm}=m_{a}^{2}+M_{i}^{2}+2 E_{a}^{(i+1)} k_{i}^{0} \pm 2 P_{a}^{(i+1)} K_{i}
$$

and the Monte-Carlo will generate the $t$ values according to

$$
t_{i}=t_{i}^{-}+r^{(i)}\left(t_{i}^{+}-t_{i}^{-}\right)
$$

The limiting values $t^{ \pm}$define the physical region boundaries of $2 \rightarrow 2$ reaction on the Chow-Low plot shown in Figure 2.

On the other hand, using a multiperipheral form in $T$ as in Eq.13, we can generate events with anisotropic behavior so as to satisfy the simulation identity [16].

$$
\int_{t_{i}^{-}}^{t_{i}} \exp \left(a_{i} t_{i}\right) d t_{i} / \int_{t_{i}^{-}}^{t_{i}^{+}} \exp \left(a_{i} t_{i}\right) d t_{i}=r^{(i)}
$$

then

$$
t_{i}=\left(1 / a_{i}\right) \ln \left\{r^{(i)}\left(\exp \left(a_{i} t_{i}^{+}\right)-\exp \left(a_{i} t_{i}^{-}\right)\right)+\exp \left(a_{i} t_{i}^{-}\right)\right\}
$$

The condition Eq.22 will spread the points in a confined Zone in the Chow-Low Plot by cutting of the high $t$ values. The parameters $a_{i}$ are directly reproduced from the comparison with experimental distributions.

\subsection{Effect of the Quark Structure}

Let use assume that the interaction takes place not with the interacting particles as a whole but rather among

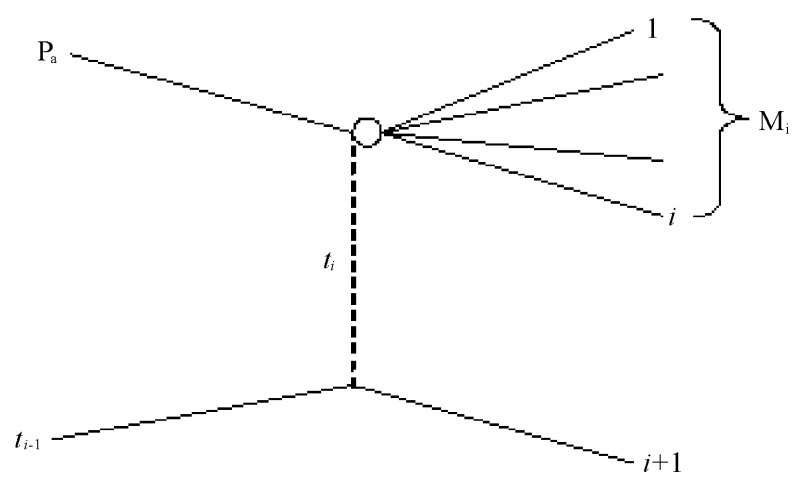

Figure 2. The basic process at stage $i$ of iteration. 
their minute constituent quarks. Neglecting the spin effect of the quark and considering, for example, $\pi p$, system as two bags containing, respectively, two and three quarks each of effective mass $m_{q}$, we assume that the reaction goes through one of the following channels:

1) One of the projectile quarks interacts with one of the target quarks. We use the symbols $(0-\infty)$ and $(0-\infty)$ to describe the two states of the first channel. The number of possible permutation of these states is 3 .

2) In the second channel, the two projectile quarks may interact with the three target quarks in a collective manner. This state is symbolized by $(0-\infty)$ ) with only one possible permutations. The square of the center-of-mass energy of each state is calculated according to;

$$
s=\left(N_{a}^{2}+N_{b}^{2}\right) m_{q}^{2}+2 m_{q} N_{a} N_{q} e_{q}
$$

where $N_{a}$ and $N_{b}$ are the number of quarks participating in the reaction from the target and projectile, respectively; $m_{q}$ is the effective quark mass and $e_{q}$ is the laboratory energy per quark. The multiplicity distribution $F(n)$ of an $n$-particle system is calculated in terms of the distribution functions of the different states of the reaction. For our example case ( $\pi p$-system), let us assume that $f_{11}(n)$ and $f_{12}(n)$ represent the phase space integrals of the state $(\mathrm{O}-\mathrm{O})$ and $(\mathrm{O}-\mathrm{O})$ ) for the first channel, so that the distribution of the first channel is obtained by a restricted superposition of the two functions;

$$
f_{1}(n)=\sum_{i} \sum_{j} Z(n)\left[\left(f_{11}(i)+f_{12}(j)\right) \delta(n-(i+j))\right]
$$

where the normalization factor, $Z(n)$ is given by

$$
Z(n)=\left[\sum_{i} \sum_{j} \delta(n-(i+j))\right]^{-1}
$$

The second channel has only one state $(0-\infty)$ represented by a phase space integral $f_{2}(n)$, then the overall distribution function $F(n)$ is

$$
F(n)=k_{1} f_{1}+k_{2} f_{2}
$$

where $k_{1}$ and $k_{2}$ are the number of possible permutations in each channel. The distribution function for any other physical quantity $x$ is simply given by

$$
H(x)=k_{1} \sum h_{1 i}(x)+k_{2} h_{2}(x)
$$

\subsubsection{Hadron-Nucleus Collision}

On extending the model to the hadron-nucleus or nucleus-nucleus collision, we follow the $N N$-base super position as expected from the features of the experimental data. We should consider the possible interactions with the nucleons forming the target nucleus $A_{t}$. The incident hadron makes successive collisions inside the target. The energy of the incident hadron (leading particle) slows down after each collision, producing a number of created hadrons each time which depends on the available energy. The phase space integral $I_{n}^{N A}$ in this case has the form;

$$
I_{n}^{N A}(s)=\sum_{v}^{A_{t}} I_{n v}\left(s_{v}\right) P\left(v, A_{t}\right) \delta\left(n-\sum_{i}^{A_{t}} n_{i}\right)
$$

where $P\left(v, A_{t}\right)$ is the probability that $v$ nucleons out of $A_{t}$ will interact with the leading particle and $I_{n v}(s v)$ is the phase space integral of $N N$ collision that produces hadrons at energy $\sqrt{s}$. The delta function in Eq.28 is to conserve the number of particles in the final state. All the nucleons are treated identically, and the $X_{N N}$ is the $N-N$ phase shift function [20,24-26]. Then, according to the eikonal approximation,

$$
p\left(l, A_{t}\right)=-\left(\begin{array}{l}
A_{t} \\
l
\end{array}\right) \sum_{j}^{l}(-1){ }^{j}\left(\begin{array}{l}
l \\
j
\end{array}\right)\left\{1-\exp \left(2 \operatorname{Re} i\left(A_{t}-l+j\right) X_{N N}\right)\right\}
$$

This approach was then worked out by putting the multi-dimension integration of Eq.16 and the generated kinematical variables into a Monte-Carlo program which was created by the author. This in turn is restored $v$ times, where $v$ is the number of collisions inside the target nucleus that is generated by a Monte-Carlo Generator according to the probability distribution Eq.29. In the first one, the incident hadron has its own incident energy and moves parallel to the collision axis $\left((Z-\right.$ axis $) \theta_{0}=0$. The output of the program determines the number of created hadrons $n_{1}$ as well as the energy $E_{1}\left(<E_{0}\right)$ and the direction $q_{1}$ of the leading particle. The leading particle leads the reaction in its second round with $E_{1}$ and $\theta_{1}$ as input parameters and creates new number of $n-2$ and so on. The number $n_{j}$ is determined according to a multiplicity generator which depends on the square of the center of mass energy $s_{j}$ in the round number $j$ :

$$
s_{j}=2 m_{N}^{2}+2 m_{N} E_{j}
$$

\section{NUCLEUS-NUCLEUS COLLISIONS}

The extension of the multi peripheral model to the nucleus-nucleus case is more complicated. The number of available collisions is multi-folded due to the contribution of the projectile nucleons. By analogy of the $N-A$ 
collision, it is possible to define the phase space integral $I_{n}^{A A}$ in $A-A$ collisions as,

$$
I_{n}^{N N}(s)=\sum_{j}^{A_{p}} \sum_{k}^{A_{t}} I_{n_{j, k}}\left(s_{j, k}\right) P_{A A}\left(j, A_{p}, k, A_{t}\right) \delta\left(n-\sum_{j, k}^{A_{t}} n_{j, k}\right)
$$

where $I_{n j, k}\left(s_{j, k}\right)$ is the phase space integral due to the knocked on nucleon number $j$ from the projectile and that, number $k$ from the target. The probability that the $A-A$ collision encounters events. So that,

$$
P_{A A}\left(j, A_{p}, k, A_{t}\right)=P\left(v_{p}, A_{p}\right) \cdot P\left(v_{t}, A_{t}\right)
$$

About 1000 events have been generated for each reaction by the Monte-Carlo according to the decay diagram of Figure 1.

\section{THEORETICAL CALCULATION}

\subsection{The Multi-Peripheral Parameters $a_{i}$ with $n$ Particle Final State}

The values of the multi-peripheral parameters $a_{i}$ play an important role in the calculation of the phase space integrals and the inclusive cross sections. The multiperipheral parameters carry all the dynamical effects that control the geometrical and kinematical behavior of the reactions. The values of $a_{i}$ are considered as fitting parameters and are determined to conserve the total energy in the center of mass of the reaction [17]. Taking all possible configurations (pairing) of quark combinations as described in Subsection 2.1.

Referring to equations Eq.12 and Eq.13 we find that the parameter $a_{n}$, plays the effective role in the dynamic matrix element which controls the generation process of the events according to the assumed number of produced particles $n_{b}$ and the square of the available energy in the center of mass $\sqrt{s}$. The parameter $a_{n}$ is just a fitting parameter in the simulation process. Its value is to conserve the energy in the generator $G(n)$.

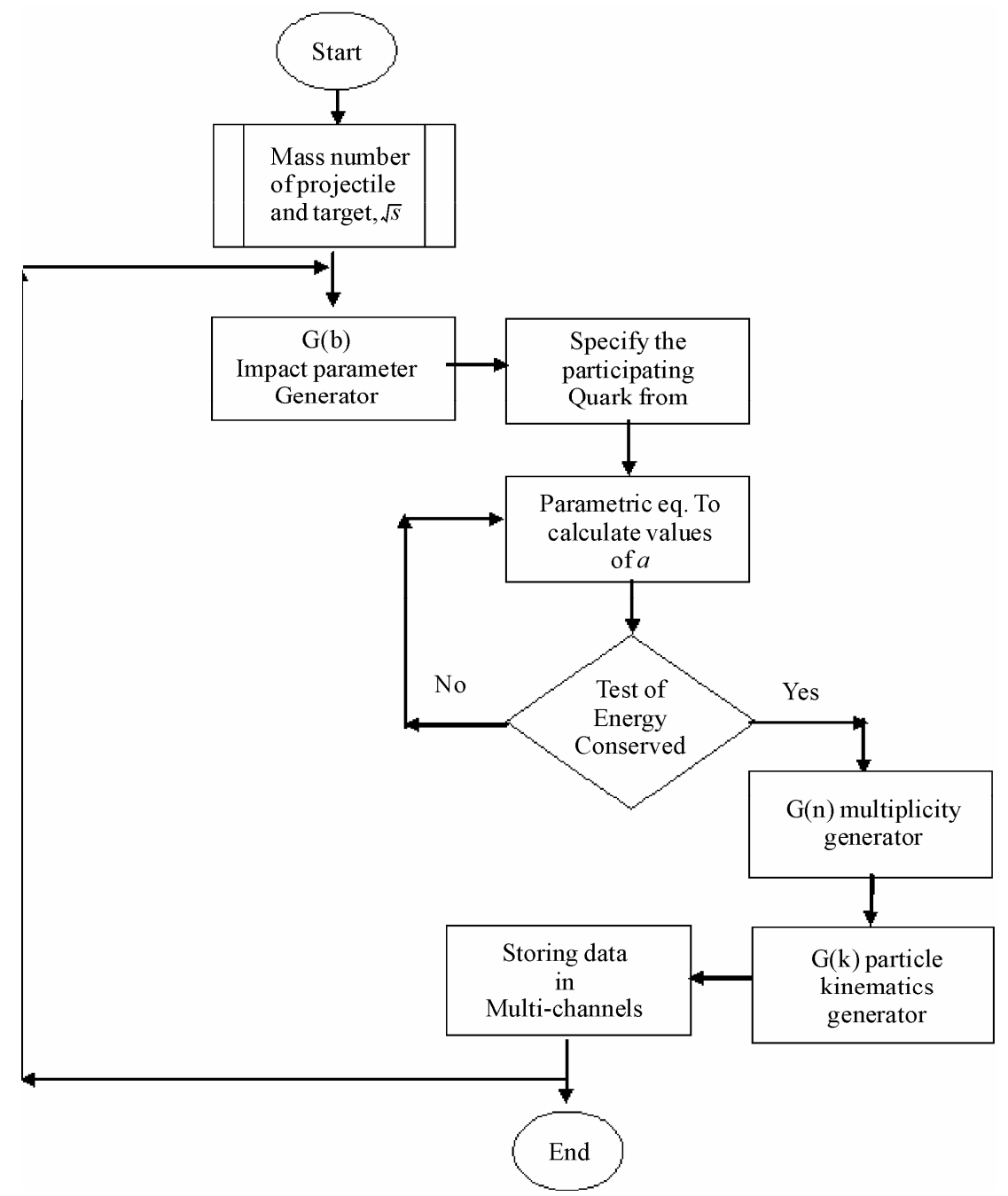

Figure 3. The flow chart of the Monte Carlo code for $p-p$ collisions. 

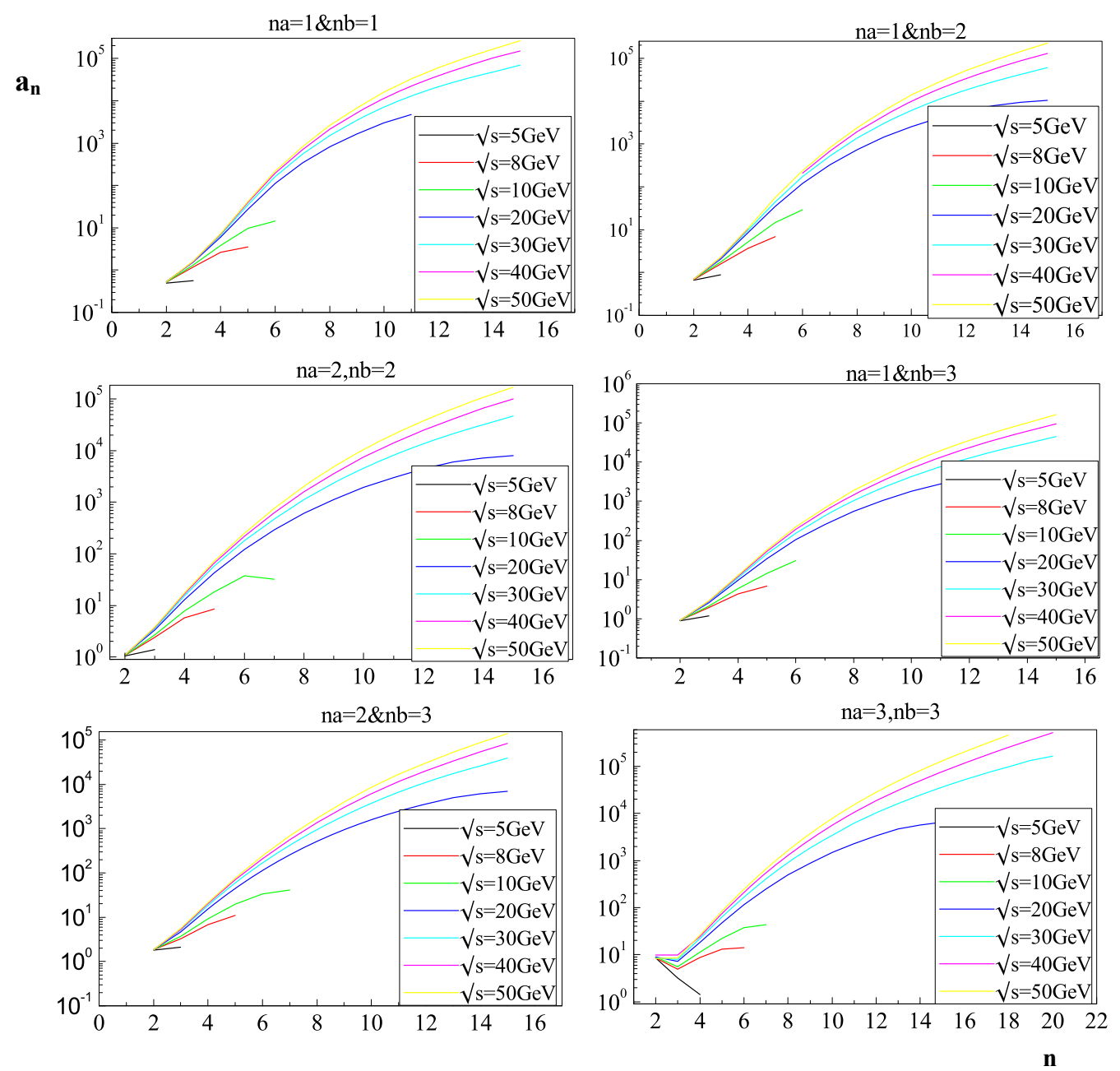

Figure 4. The multi-peripheral parameter (a) deduced for the $n$-particle final state in case of proton -proton collisions.

In Figure 4 we display the values of the multi-peripheral parameters $a_{n}$ as a function of number of created particles $\mathrm{n}$ in the final state at different center of mass energies, $\sqrt{s}=5,8,10,20,30,50 \mathrm{GeV}$, for different configurations of participating nucleons from projectile $a_{n}$ and target $n_{b}$. In all cases the value of $a$ n increases in general with $n$ and $\sqrt{s}$. The relation of $a_{n}$ with $n$ and $\sqrt{s}$ is parameterized in a polynomial form to speed up the simulation process of the generator.

A Monte-Carlo code is designed to generate $(p p)$ events at incident energies of 8.8, 102 and $400 \mathrm{GeV}$. Figure 3 shows the flow chart of the code generators. We start with the initial incident energy. The projectile and target protons consist of 3-quarks for each. The following generators are considered:

1) -Impact parameter generator $G(b)$ to generate the value of the impact parameter according to simple geometrical aspects.

2) -Specifying the target and projectile number of quark participating in the collision according to the impact parameter value.

3) -Multiplicity Generator G(n) to generate the number of particles in the final state.

4) -The kinematics generator $\mathrm{G}(\mathrm{k})$ to generate particle kinematics in the final state according to the Feynman binary diagram Figure 2.

5) -Combining the possible number of quarks that participate in the reaction

6) -Storing the kinematical data in multi-channels of momentum-angular and energy spaces.

7) -END.

In dealing with the proton-nucleus $(p A)$ and the nucleus-nucleus $(A A)$ collisions we considered the Monte-Carlo code of $(p p)$ as a subroutine in a more general code. It is assumed that a number of $v$-binary collisions of $(p p)$ type would be carried out inside the $(p A)$ or $(A A)$ collision. Consequently, the $p p$ code is folded $v$-times for each $(A A)$ event. The number $v$ depends mainly upon the effective target mass at the considered impact parameter. 
The Monte-Carlo code is run to the case of $p-C$ collision at $200 \mathrm{GeV}$. All possible values of a projectile nucleon participant in the reaction are considered. The case of $n_{p}=1$ and $n_{t}=1$ refers to the single nucleon-nucleon collision. It rather happened for the conditions of peripheral interactions. As collision orients towards the central collision, more target nucleons contribute to the reaction. Figure 5(a) Shows that the shower particle production (created particles) increases with increasing the number of target participant nucleons; where the available center of mass energy increases. The multiplicity distribution of the shower created particles may fit a Gaussian distribution, the peak and the dispersion of which shifts forward as $n_{t}$ increases. The contribution of each $n_{t}$ value has a certain weight factor that is related to the impact parameter weight. Averaging over all possible values of the impact parameters results in the overall multiplicity distribution that is displayed in Figure 5(b). It was found that the average value of the charged particle multiplicity is 4.35 and the dispersion is 1.37557 for the $p$ - $C$ interaction at $200 \mathrm{GeV}$. The same procedure is carried out for the He-Be interaction as an example of $(A A)$ collision at the same incident energy for the sake of comparison. In this case both the number of projectile and target participant nucleons $n_{p}$ and $n_{t}$ have appreciable effect in shaping the charged particle multiplicity distributions. The number $n_{p}$ plays the role of multiplication factor in the production process.

In Figure 6 we demonstrate the family of curves representing the results of multiplicity distributions for the

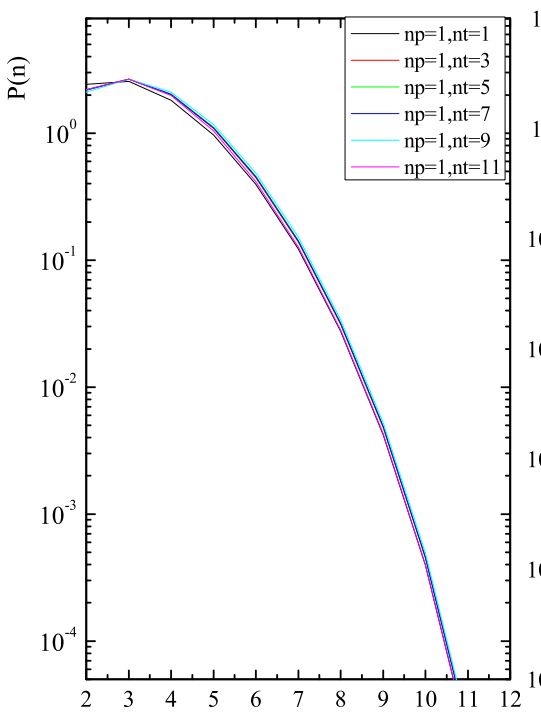

(a) case where all projectile nucleons participate the reaction $n_{p}=4$ while parts of the target contribute as $n_{t}=$ 4, 5, 6, 7 and 8 .

\section{RESULTS AND DISCUSSION}

The numerical computation of the charged and negative charged multiplicity distribution of the outgoing particles in $(p-p)$ interactions at $8.8 \mathrm{GeV}$ Figure 7, $102 \mathrm{GeV}$ Figure 8, $400 \mathrm{GeV}$ Figure 9, $(p-d)$ interaction at $28 \mathrm{GeV}$ Figure 10 and $(\mathrm{He}-\mathrm{He})$ interaction at $120 \mathrm{Gev}$ Figure 11 are calculated. A Monte-Carlo program designed by the authors is used to simulate about 1000 events for each final state of specific $n$-values. The multi-peripheral matrix element is used according to Eq.12 to calculate the phase space integral and the production cross section. The cut-off boundaries $t_{i}^{ \pm}$of the physical region is used according to Eq.22.

The proposed model is a statistical model in its nature. It assumes a large phase space and consequently large number of quantum states to work in a relevant environment.

Figures 7-11 show that the prediction of the model comes closer to the experimental data as simple as increasing both the available energy and the number of interacting particles (the size of the target and the projectile nuclei) that meets with the increase of the volume of phase space.

Table 1 shows the Chi-square values for the reaction under consideration to test the validity or the behavior of the model against the projectile-target size and the energy of the reaction.

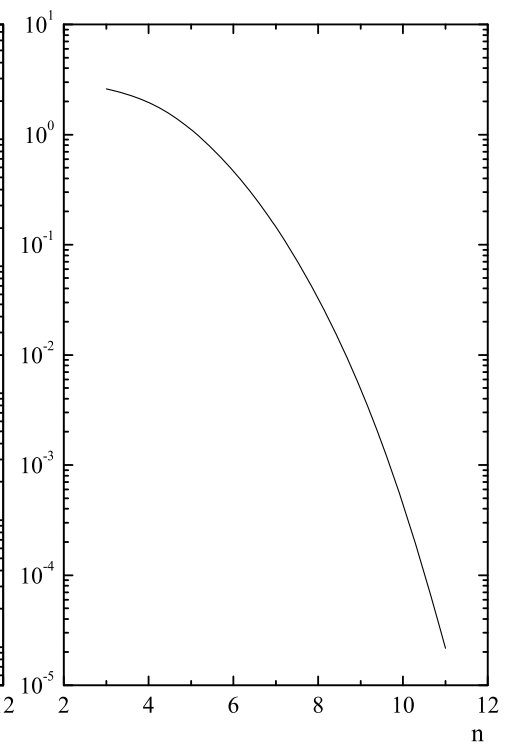

(b)

Figure 5. (a) Multiplicity distributions of the produced particles for $200 \mathrm{GeV}$ proton-Carbon $p$-C interactions for different number of target participants; (b) Total multiplicity distribution of the produced particles for $200 \mathrm{GeV} p$-C interactions. 


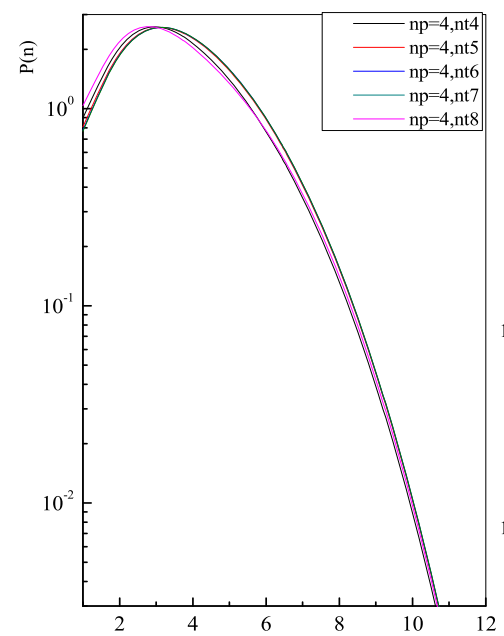

(a)

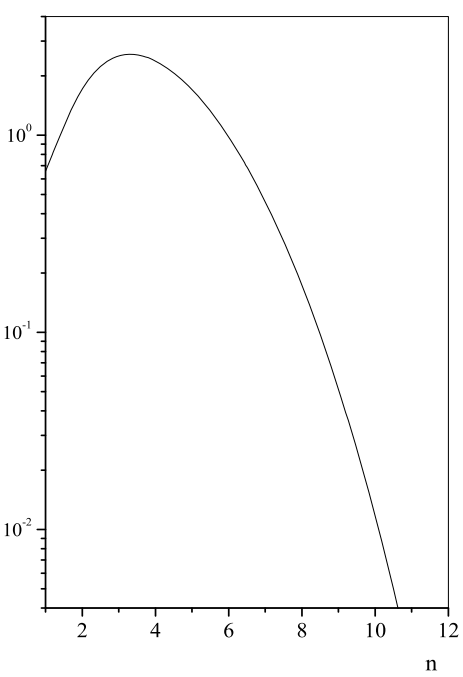

(b)

Figure 6. (a) Multiplicity distributions of the produced particles in $\mathrm{He}-\mathrm{Be}$ interactions at $200 \mathrm{GeV} / \mathrm{n}$ for different target participants; (b) Total multiplicity distributions of the produced particles in Helium-Brelium $\mathrm{He}-\mathrm{Be}$ interactions at $200 \mathrm{GeV} / \mathrm{n}$.

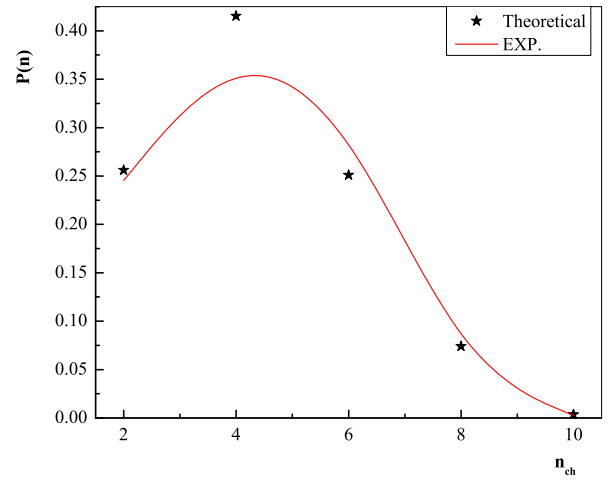

Figure 7. The multiplicity distributions of charged particles produced in $\mathrm{p} \_\mathrm{p}$ interactions at $8.8 \mathrm{GeV}$.The red curve is the model prediction, the black stars are the experimental data which have been taken from [27].

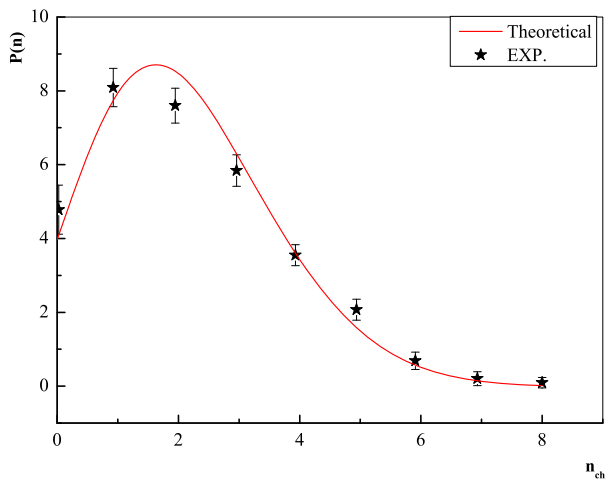

Figure 8. The multiplicity distributions of negative charged particles produced in $p$ - $p$ interactions at 102 $\mathrm{GeV}$. The red curve is the model prediction, the black stars with error bars are the experimental data which have been taken from [28].

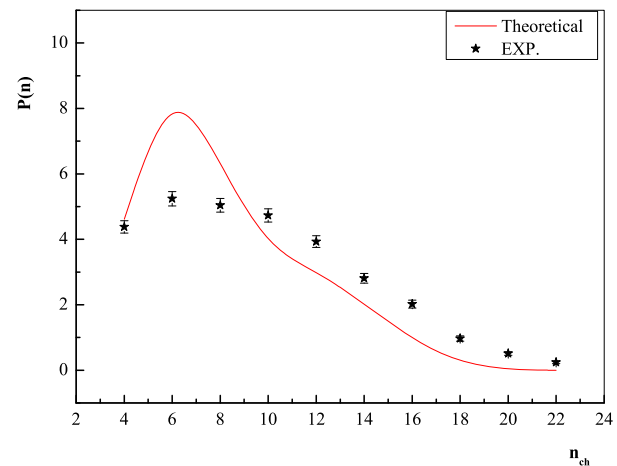

Figure 9. The multiplicity distributions of negative charged particles produced in $p$ - $p$ interactions at $400 \mathrm{GeV}$. The red curve is the model prediction, the black stars with error bars are the experimental data which have been taken from [29].

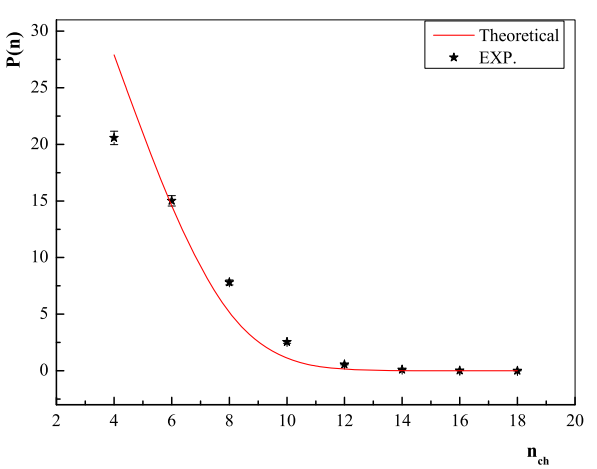

Figure 10. The multiplicity distributions of charged particles produced in $p$ - $d$ interactions at $28 \mathrm{GeV}$. The red curve is the model prediction. The black stars are the experimental data which have been taken from [30]. 


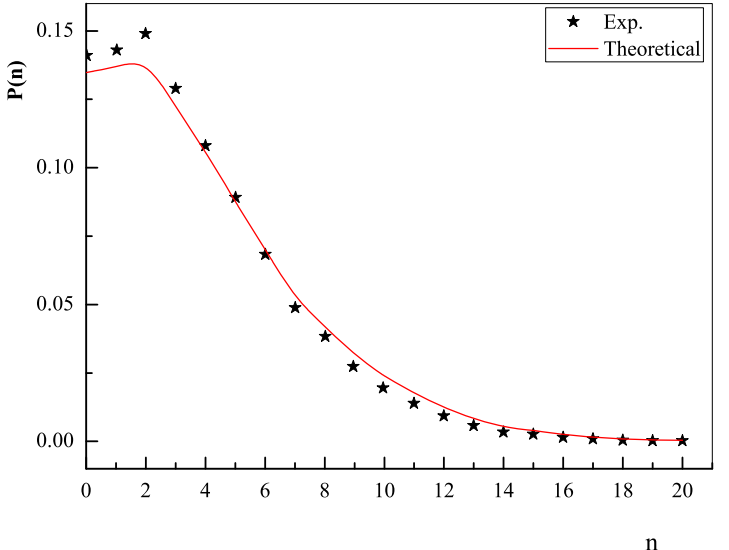

Figure 11. The multiplicity distribution of the particles produced in $\mathrm{He}-\mathrm{He}$ interactions at $120 \mathrm{GeV}$. The red curve is the model prediction. The black stars are the experimental data which have been taken from [31].

Table 1. The Chi-square of the reactions.

\begin{tabular}{ccc}
\hline Reaction & Energy & Chi-Square \\
\hline$p-p$ & $8.8 \mathrm{GeV}$ & 0.005066 \\
$p-p$ & $102 \mathrm{GeV}$ & 0.004561 \\
$p-p$ & $400 \mathrm{GeV}$ & 0.822473 \\
$p-d$ & $28 \mathrm{GeV}$ & 0.034502 \\
$\mathrm{He}-\mathrm{He}$ & $120 \mathrm{GeV}$ & 0.000382 \\
\hline
\end{tabular}

\section{CONCLUSIONS}

The multi-peripheral model is extended to the nucleon-nucleus and the nucleus-nucleus interaction on the basis of nucleon-nucleon collisions, where the phase space integral of the nucleon-nucleon and nucleus-nucleus interaction is folded several times according to the number of encountered nucleons from the target. The number of created particles in each collision is summed over to get the production in the nucleon-nucleon case, where the conservation of number of particles in the final state is taken into consideration. The inclusive cross section is calculated and showed a fair agreement with experimental data.

\section{REFERENCES}

[1] Bjorken, J.D. (1983) Principles of opticsoo. Physical Review D, 27, 140.

[2] Busza, W. and Goldhaber, A. (1984) Nuclear stopping power. Physics Letters B, 139(4), 235-238.

[3] Barton, D.S., et al. (1983) Experimental-study of the A-dependence of inclusive hadron fragmentation. Physical Review D, 27(11), 2580-2599.

[4] Andersson, B., Gustafson, G. and Nilsson-Almqvist, B. (1987) A model for low-pT hadronic reactions with generalizations to hadron-nucleus and nucleus-nucleus collisions. Nuclear Physics B, 281(1-2), 289-309.

[5] Nilsson-Almqvist, B. and Stenlund, E. (1987) Interac- tions between hadrons and nuclei: The Lund Monte Carlo - FRITIOF version 1.6. Computer Physics Communications, 43(3), 387-397.

[6] Sorge, H., Von Keitz, A., Mattiello, R., Stocker, H. and Greiner, W. (1990) Energy density, stopping and flow in 10-20 A GeV heavy ion collisions. Physics Letters B, 243, 7-12.

[7] Mattiello, R., et al. (1991) Multistrangeness production in heavy ion collisions. Nuclear Physics B, 24(2), 221-233.

[8] Wang, X.N. and Gyulassy, M. (1991) HIJING: A Monte Carlo model for multiple jet production in $p p, p A$, and AA collisions. Physical Review D, 44, 3501-3516.

[9] Geiger, K. and Muller, B. (1992) Dynamics of parton cascades in highly relativistic nuclear collisions. Nuclear Physics A, 544(2), 467-470.

[10] Geiger, K. (1992) Thermalization in ultrarelativistic nuclear collisions I. Parton kinetics and quark-gluon plasma formation. Physical Review D, 46(11), 4965.

[11] Geiger, K. (1993) Particle production in high energy nuclear collisions: Parton cascade - cluster hadronization model. Physical Review D, 47, 133.

[12] Brenner, A.E., et al. (1982) Experimental study of single-particle inclusive hadron scattering and associated multiplicities. Physical Review D, 26, 1497-1553.

[13] Amati, D., Stanghellini, A. and Fubini, S. (1962) Theory of high-energy scattering and multiple production. Nuovo Cimento, 26, 896-954.

[14] Hassan, N.M. (2007), Charged particle multiplicity produced in neutrino-nucleon interactions at high energies. Scientific Research and Essays, 2(6), 171-176.

[15] Hussein, M.T., Hassan, N. M. and Rabea, A. (1994) ICTP, Trieste, internal report IC/93/111. Nuovo Cimento $A, \mathbf{1 0 7}, 749$.

[16] Hussein, M.T. (1993) ICTP, Trieste, internal report IC/92/166. Nuovo Cimento A, 106, 481.

[17] Byckling, E. and Kajantie, K. (1973) Particle kinematics. John Wiley and Sons, New York, 190.

[18] Hussein, M.T., Hassan, N.M. and Rabea, A. (1994) Hadron fragmentation at high energies. Particle World, 4(1), 4.

[19] Hussein, M.T., Hassan, N.M. and Allam, M.A. (1997) A Multi-cluster model in hadron nucleus interactions at high energies. Turkish Journal of Physics, 21, 241.

[20] Hegab, M.K., Hussein, M.T. and Hassan, N.M. (1990) Nucleus-nucleus collisions at high energies. Zeitschrift für Physik A, 336, 345.

[21] Hussein, M.T. (1996) Monte Carlo simulation for hadron fragmentation at high energies. Invited Talk, First International Conference on Basic Science and Advanced Technology, Assiut, 9-12 November 1996.

[22] Hussein, M.T., Rabea, A., El-Naghy, A. and Hassan, N.M. (1995) A string model for hadron interactions at high energies. Progress of Theoretical Physics Japan, 93(3), 585 .

[23] Hassan, N.M., El-Harby, N. and Hussein, M.T. (2000) A Monte Carlo generator for high energy nucleus-nucleus collision. Acta Physiologica Academiae Scientiarum Hungaricae, 12, 33-46.

[24] Meggiolaro, E. (1998) High-energy quark-quark scattering and the eikonal approximation. Nuclear Physics BProceedings Supplements, 64, 191.

[25] Pereira, F. and Ferreira, E. (1999) Eikonal profile func- 
tions and amplitudes for $\mathrm{pp}$ and $\mathrm{p}^{-} \mathrm{p}$ scattering. Physical Review D, 59(1).

[26] Ducati, M.B.G., Goncalves, V.P. (1999) The AGL equation from the dipole picture. Nuclear Physics B, 557(1), 296-306.

[27] Booth, C.N., Ansorge, R.E. and Carter, J. (1983) Differences between $\mathrm{p}^{-} \mathrm{p}$ and $\mathrm{pp}$ interactions at $8.8 \mathrm{GeV} / \mathrm{c}$ and their relationship to $\mathrm{p}^{-} \mathrm{p}$ annihilations. Physical Review D, 27, 2018-2040.

[28] Aznauryan, I.G. and Ter-Isaakyan, N.L. (1980) Anomalous magnetic-moments of quarks in magnetic dipole transitions of hadrons. Soviet Journal of Nuclear Physics,
31(6), 871-875.

[29] Kass, R.D., Ko, W., Lander, R.L., et al. (1979) Chargedand neutral-particle production from $400-\mathrm{GeV} / \mathrm{c}$ pp collisions. Physical Review D, 20(3), 605-614.

[30] Hanlon, J., Panvini, R.S., Walters, J.W., Webster, M.S. and Morris, T.W. (1979) Multiplicity distributions in $28-\mathrm{GeV} / \mathrm{c}$ pd interactions and double scattering in deuterium. Physical Review D, 19(1), 49-54.

[31] Akesson, T., Albrow, M.G., Almehed, S., et al. (1982) Multiplicity distributions in $p-\alpha$ and $\alpha-\alpha$ collisions in the CERN ISR. Physics Letters $B, \mathbf{1 1 9}, 464$. 